\title{
Pacemaker lead infection: report of three cases and review of the literature
}

\author{
J G Voet, Y R Vandekerckhove, L L Muyldermans, L H Missault, L J Matthys
}

Department of Cardiology, AZ Sint-Jan, Ruddershove 10, 8000 Brugge, Belgium J G Voet Y R Vandekerckhove L L Muyldermans

L H Missault

L J Matthys

Correspondence to: Dr Vandekerckhove.

Accepted for publication 7 July 1998

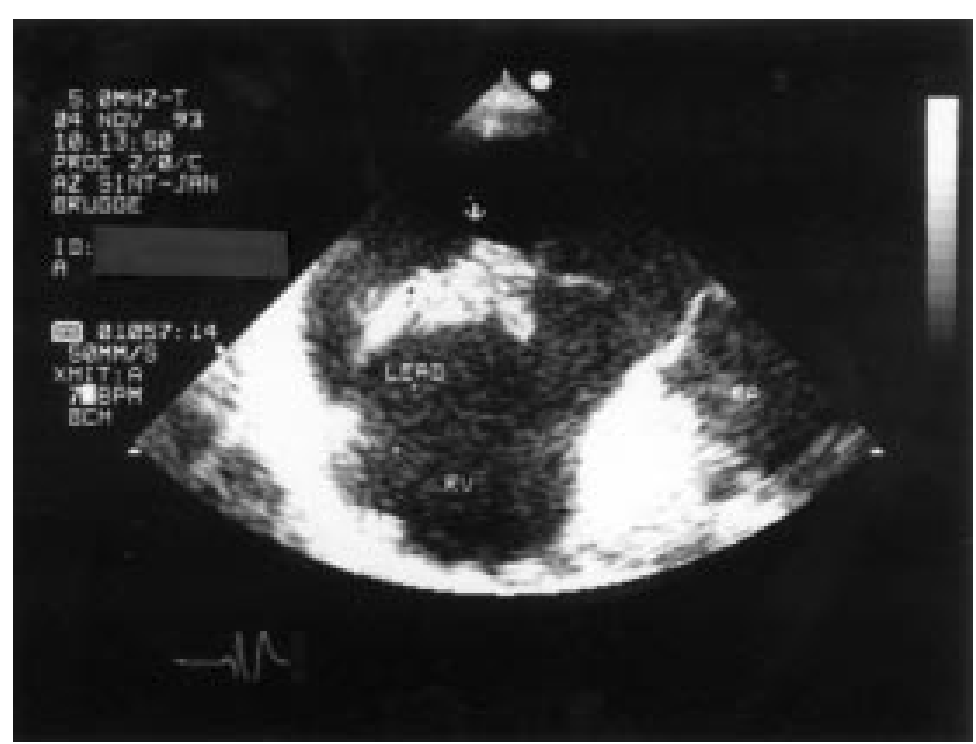

Figure 1 Transoesophageal echocardiogram of patient 1 showing a vegetation of

$32 \times 12 \mathrm{~mm}$ attached at the ventricular lead. $R V$, right ventricle; $R A$, right atrium.

\begin{abstract}
Pacemaker lead infection is a rare condition, most often occurring when intervention is needed after pacemaker implantation. Diagnosis is by blood cultures and confirmation by transoesophageal echocardiography; transthoracic echocardiography is often inadequate. A literature review indicated the microorganism most responsible for late lead infection is Staphylococcus epidermidis (which can grow on plastic material). A retrospective analysis of patient files from the authors' institution (1993-97) yielded three patients with proven pacemaker lead endocarditis. The diagnosis of pacemaker endocarditis was by transoesophageal echocardiography. The endocarditis appeared after a long period and in two of the three patients there was $S$ epidermidis infection. Thoracotomy with removal of the infected system was performed because of the large dimensions of the vegetations. A new pacemaker was implanted: in one patient with endocardial leads, in the other two with epicardial leads. All three patients recovered well and follow up was uneventful for at least one year.
\end{abstract} (Heart 1999;81:88-91)
Keywords: endocarditis; pacemaker lead infection; staphylococci; transoesophageal echocardiography

Pacemaker endocarditis is life threatening and an early diagnosis is important. At the end of 1996, two patients with pacemaker lead infection presented at our institution. Review of patient files (1993-97) revealed a third case of proven pacemaker lead infection.

\section{Case reports}

PATIENT 1

Patient 1 was a 77 year old woman with a euthyroid multinodular struma. In 1990 she received a DDD pacemaker in another hospital because of sick sinus syndrome with intermittent AV block. In 1991 there was a malfunction of the pacemaker with cardiovascular collapse, followed by ventricular lead extraction and a replacement with a similar lead at the same intervention. The pacemaker was programmed in VVI mode.

One year later the patient had a pocket infection with penicillin sensitive Staphylococcus epidermidis. At that time the useless atrial pacemaker lead was removed. Another year later the patient was admitted several times because of fever and came finally to our department for further investigation. Blood cultures were positive for penicillin resistant $S$ epidermidis. Transthoracic echocardiography showed a mobile mass but transoesophageal echocardiography showed a large mobile mass $(32 \times 12 \mathrm{~mm})$ attached at the pacemaker lead (fig 1).

The differentiation has to be made between a thrombus and a vegetation. Endocarditis was suspected and treatment with high dose antibiotics started. Because of recurrent septicaemia, and despite her age, the patient was referred for thoracotomy with removal of the whole pacemaker system. She was given an epicardial pacemaker and six weeks' postoperative treatment with antibiotics. Follow up of three years was uneventful.

PATIENT 2

Patient 2 was a 40 year old man who received a DDD pacemaker via the right cephalic vein in April 1996, because of recurrent syncope from sinus arrest. One month later, an unacceptable threshold in the ventricle was detected and a 
new intervention with reposition of this lead was necessary.

Four months later he had a high fever $\left(39.6^{\circ} \mathrm{C}\right)$ and was treated empirically by his family physician. Six months after the initial implantation he was admitted to our hospital because of persisting high fever.

Transoesophageal echocardiography showed a large vegetation $(19 \times 16 \mathrm{~mm})$ attached to the ventricular pacemaker lead (fig 2). Heart valve function was normal.

Blood cultures were positive for $S$ epidermidis. $\mathrm{He}$ was treated initially with high dose intravenous antibiotics and then a thoracotomy was performed. After six weeks of antibiotic treatment a new transvenous DDD pacemaker was implanted via the left cephalic vein. Follow up of one year was uneventful.

PATIENT 3

Patient 3 was a 74 year old woman with diabetes. She had intermittent auricular fibrillation, an earlier cerebrovascular accident, arterial hypertension, and hyperlipidaemia.

In 1994 a DDDR pacemaker was implanted because of extreme bradycardia and dizziness. Two days later, a new intervention needed to be performed because of lead displacement.

In 1995 she was in an accident and suffered multiple rib fractures and left haemothorax. She recovered well but in November 1996 a malfunction of the atrial lead was noted and a switch to VVI mode was needed.

Two years after implantation the patient was admitted with cold hyperdynamic sepsis and threatening multiple organ failure. There was also a malfunction of the pacemaker with $2: 1$ exit block. Transoesophageal echocardiography showed multiple lead vegetations and severe mitral (3/4) and tricuspid (4/4) regurgitation. A thoracotomy was performed, the pacemaker system removed, and epicardial leads implanted. A tricuspid annuloplasty was performed at the same time. Blood cultures were negative (antibiotic treatment was started before admission).

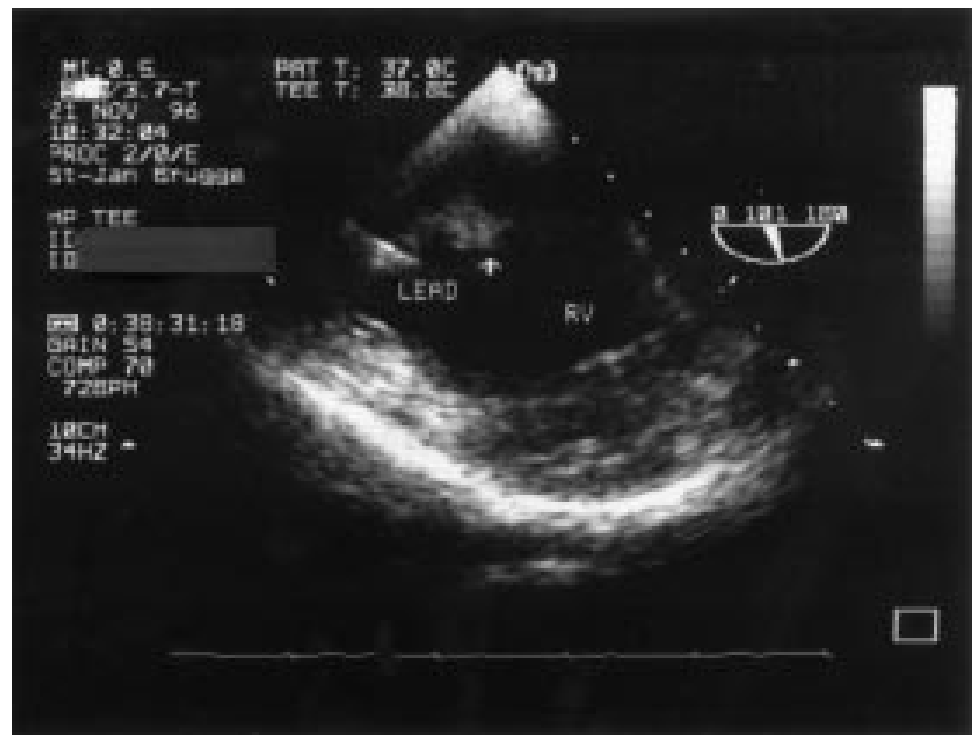

Figure 2 Transoesophageal echocardiogram of patient 2 showing a vegetation of $19 \times 16 \mathrm{~mm}$ attached at the ventricular lead. RV, right ventricle.

\section{Discussion}

The reported incidence of infection of the permanent pacemaker system is between $0.3 \%$ and $12.6 \%$. This may involve infection of the generator pocket (appearing two to five weeks after implantation) or infection of the electrodes (appearing after an average of 33 weeks) and may be associated with bacteraemia, with or without concomitant infective endocarditis. Documented septicaemia is rare $(1-3 \%) \cdot{ }^{1-3}$ To the best of our knowledge the incidence of pacemaker endocarditis has not been reported.

Septicaemia is diagnosed when at least two haemocultures are positive or when one culture is positive together with at least one clinical manifestation of septicaemia (fever, chills, raised white blood cell count, sedimentation).

Review of the literature presents some predisposing factors for pacemaker infection. As in our three cases, repeated surgical procedures on the pacemaker system is the most important and these are responsible for 66$73 \%$ of the infections. The incidence of infection decreases with a higher degree of aseptic care. Older series noted that the highest rate occurs when implantation is performed in the $x$ ray department or angiography unit rather than the operating room. ${ }^{4}$ General recommendations now allow implantation in the angiography or electrophysiology unit, as long as strictly aseptic conditions are present. ${ }^{5}$ Other predisposing factors for lead infection are postimplantation pocket haemorrhage, erosis, necrosis or infection. Some systemic factors contributing to a higher incidence are diabetes mellitus, a thin skin, the use of corticosteroids, age, intravenous catheters, neoplasm, the use of anticoagulants, temporary pacing, dermatological diseases, and other infectious foci. ${ }^{164}$

Early diagnosis of a pacemaker lead infection is difficult. One should be suspicious in the case of a patient with a pacemaker with persisting bacteraemia or fever without another focus of infection. Endocarditis should be considered until proven otherwise.

The use of transoesophageal echocardiography is becoming increasingly useful as a diagnostic technique and it seems to be much more sensitive in identifying left side endocarditis and lead vegetations than the transthoracic view. ${ }^{1267}$ There are three reasons why the transthoracic approach is less valuable:

- inadequate precordial acoustic window

- the pacemaker lead produces reverberations

- the transoesophageal approach reveals a better view on the right atrium and the superior vena cava.

One study ${ }^{6}$ suggests that transoesophageal echocardiography is not superior to transthoracic echocardiography in detecting right sided endocarditis in intravenous drug abusers. Vilacosta and colleagues ${ }^{8}$ showed that in patients with the clinical suspicion of an infected permanent transvenous pacemaker, transoesophageal echocardiography is superior in detecting lead vegetations. Transthoracic echocardiography detected vegetations in only two 
patients whereas transoesophageal echocardiography demonstrated them in seven of 10 patients. In a prospective study of 23 patients, ${ }^{9}$ transoesophageal echocardiography was positive in 21 and its use contributed to early diagnosis.

Pacemaker lead infection is a life threatening situation. Haematogenous dissemination can produce relapsing septic episodes, pulmonary symptoms from emboli and involvement of the tricuspid valve leading to regurgitation or, rarely, stenosis. ${ }^{10}$ Treatment of an infected pacemaker system depends on the knowledge of the clinical course and microbiological features. Most pacemaker lead infections are caused by staphylococci ${ }^{111}$; other microorganisms may also be responsible (such as corynebacterium, enterococci). Early infections after implantation tend to be caused by Staphylococcus aureus, whereas late infections are caused by $S$ epidermidis. ${ }^{71}$ One can imagine that microorganisms can grow on native tissue, but it is hard for them to survive on plastic lead surfaces. It has been shown ${ }^{12}$ that staphylococci are able to adhere to plastic surfaces, to grow on plastic material (with the possibility of using plastic components for growth), and to produce an extracellular amorphous material (slime).

Peters and colleagues ${ }^{12}$ have shown, in vitro and in vivo, that some staphylococci can grow on the plastic sheath of the pacemaker wire without external supplies. They produce a slimy amorphous material that may be important in the staphylococcal defence against host defence mechanisms and antibiotic treatment, and in the maintenance of colonisation. This may explain why lead infections are hard to manage conservatively and lead extraction is virtually always advisable.

A complete removal of all the foreign material is suggested when pacemaker system infection occurs, ${ }^{3711}$ whether it is endocarditis or an obvious pocket infection. Two techniques can be used ${ }^{2}{ }^{13}{ }^{14-16}$ :

- surgical removal by thoracotomy and extracorporeal circulation

- percutaneous removal by constant traction or intravascular extraction techniques.

It has been shown that in the hands of experienced operators, with appropriate precautions and patient selection, the intravascular extraction technique allows the removal of up to $98 \%$ of intravascular leads. The chosen technique depends on the size of the vegetation on transoesophageal echocardiography, the alteration of the tricuspid valve, and the general condition of the patient. It is recommended to remove vegetations of more than $10 \mathrm{~mm}$ by thoracotomy, as we have done in our patients. Implantation of a new pacemaker can be safely done during the same operation. There is no need for two stage intervention with interim temporary pacing.

The mortality in pacemaker endocarditis is high. A 1994 report concerning 44 Israeli patients with a documented pacemaker endocarditis noted an overall mortality of $24 \% .{ }^{17}$ Mortality was $20 \%$ in the patients who had the infected pacemaker wire surgically removed, compared to $31 \%$ in those treated with antibiotics alone.

The mortality in other series varied in the surgical group from $0-28 \%$ (mean 13\%) and in the conservative group from $0-40 \%$ (mean $32 \%) .{ }^{17}$

Prevention is better than cure and we recommend the following:

- if temporary pacing is necessary, it should be as short in time as possible

- implantation should be done in the best aseptic conditions

- no more people should be in the room than necessary to perform the implantation

- good surgical haemostasis should be enhanced; try to avoid postoperative drainage

- prophylactic antibiotics has been recommended but is not routine.

\section{CONCLUSION}

We report our experience in management of pacemaker lead infection. This is a rare but life threatening condition. It can be prevented by strict aseptic circumstances. The diagnosis must be considered in patients with fever, positive blood cultures (especially staphylococci) or septicaemia and a transvenous permanent pacemaker. It has been shown that transoesophageal echocardiography is very helpful in the diagnosis. The results of several series indicate it is best to remove the whole pacemaker system by lead extraction or thoracotomy when endocarditis or septicaemia is present.

In our three cases, the diagnosis of pacemaker endocarditis was made by transoesophageal echocardiography. The endocarditis appeared after a long period and in two of the three patients we found $S$ epidermidis infection, consistent with the literature. We performed a thoracotomy with removal of the infected system because of the large dimensions of the vegetations. A new pacemaker was implanted: in patient 1 with endocardial leads, in the other two patients with epicardial leads. All three patients recovered very well and follow up was uneventful for at least one year.

1 Vilacosta I, Zamorano J, Camino A, et al. Infected ransvenous permanent pacemakers: role of transoesophageal echocardiography. Am Heart $\mathcal{F}$ 1993;125:904-6.

2 Zehender M, Büchner C, Geibel A, et al. Diagnosis of hidden pacemaker lead sepsis by transoesophageal echocardiography and a new technique for lead extraction. Am Heart $\mathcal{f} 1989 ; 118: 1050-2$.

3 Choo MH, Holmes DR Jr, Gersh BJ, et al. Permanent pacemaker infections: characterization and management. Am $\mathcal{F}$ Cardiol 1981;48:559-63.

4 Goldman BS, Macgregor D. Management of infected pacemaker systems. Clin Prog Pacing Electrophysiol 1984;2:22035 .

5 Daubert JC, Lévy S, Medvedowsky JL. Recommendations sur les bonnes pratiques dans les techniques intracavitaires de diagnostic et de traitement des arythmies cardiaques. Arch Mal Coeur 1994;87:1213-24.

6 San Roman JA, Vilacosta I, Zamorano J, et al. Transoesophageal echocardiography in right-sided endocarditis. 7 Am Coll Cardiol 1993;21:1226-30.

7 Klug D, Lacroix D, Savoye C, et al. Systemic infection related to endocarditis on pacemaker leads. Circulation 1997;95:2098-107.

8 Vilacosta I, Sarria C, San Roman JA, et al. Usefulness of transoesophageal echocardiography for diagnosis of infected transvenous permanent pacemakers. Circulation 1994;89:2684-7.

9 Victor F, De Place C, Camus C, et al. Transoesophageal echocardiographic features of pacemaker lead infection: a prospective study [abstract]. PACE 1997;20(suppl):1153. 
10 Enia F, Mauro RL, Meschisi F, et al. Right-sided infective endocarditis with acquired tricuspid valve stenosis associated with transvenous pacemaker: a case report. $P A C E$ 1991;14:1093-7.

11 Bluhm G, Jolander I, Levander-Lindgren $M$, et al. Septicemia and endocarditis: uncommon but serious complications in connection with permanent cardiac pacing. Scand $\mathcal{F}$ Thorac Cardiovasc Surg 1987;16:65-70.

12 Peters G, Saborowski F, Locci R, et al. Investigations on staphylococcal infection of transvenous endocardial pacemaker electrodes. Am Heart f 1984;108:359-65.

13 Furman S. Pacemaker infection [editorial]. PACE 1986;9:
14 Colavita PG, Zimmern SH, Gallagher JJ, et al. Intravascular extraction of chronic pacemaker leads: efficacy and followup. PACE 1993;16:2333-6.

15 Smith HJ, Fearnot NE, Byrd CL, et al. Five-years experience with intravascular lead extraction. PACE 1994; 17:2016-20.

16 Niederhäuser U, von Vegesser LK, Carrel TP, et al. Infected endocardial pacemaker electrodes: successful open intracardiac removal. PACE 1993;16:303-8.

17 Arber N, Pras E, Copperman Y, et al. Pacemaker endocarditis. Report of 44 cases and review of the literature. Medicine 1994;73:299-305.

\section{IMAGES IN CARDIOLOGY}

\section{Eustachian valve endocarditis diagnosed by transoesophageal echocardiography}

A 75 year old woman who had been paced with a VVI system some years earlier underwent an elective incisional hernia repair and sigmoidoscopy. Her postoperative course was initially uncomplicated but on day 6 she developed spiking temperatures and rigors accompanied by paroxysms of hypoxia. Examination confirmed known mitral regurgitation. There were no signs of endocarditis. Chest radiography and ventilation/perfusion scan were unremarkable. Transthoracic echocardiography was un-

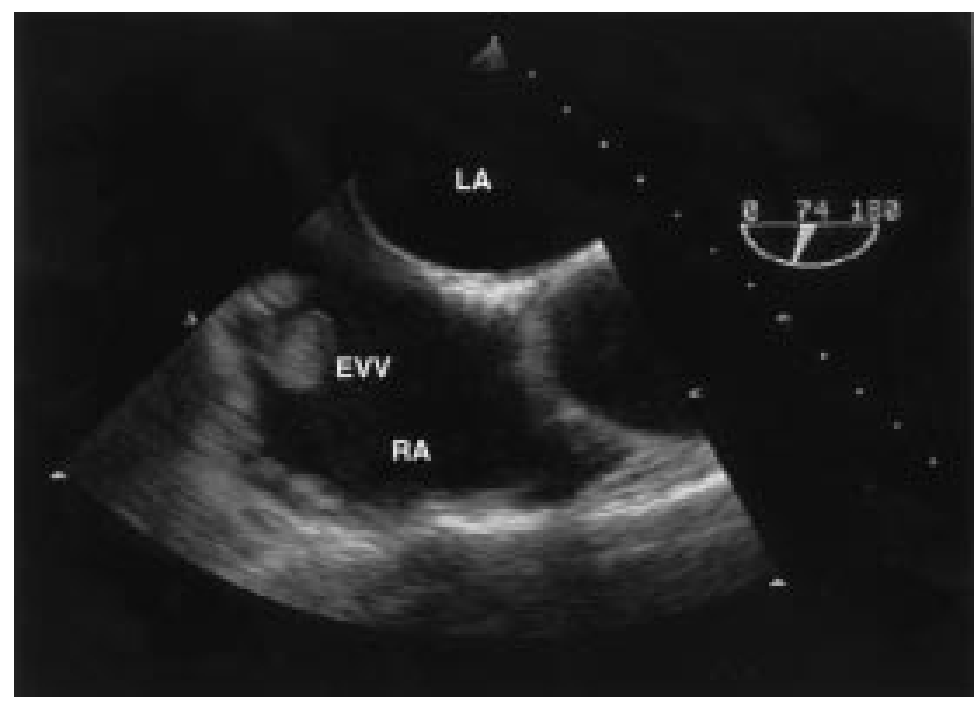

changed from a previous study with normal $\mathrm{A}-\mathrm{V}$ and semilunar valves, and mild mitral regurgitation. Blood cultures grew Enterobacter cloacae.

The triad of pyrexia, hypoxic paroxysms, and positive blood cultures strongly suggested right sided endocarditis, and transoesophageal echocardiography was performed. This revealed a $1.3 \mathrm{~cm}^{2}$ highly mobile vegetation adherent to the eustachian valve. There was no evidence of endocarditis elsewhere. The patient responded well to intravenous antibiotics, with full resolution of the vegetation.

The eustachian valve is a remnant of the right sinus venosus valve, which is vestigial in the adult heart where it is highly variable morphologically, ranging from absence to a long thread-like, fenestrated structure or Chiari's network. All five previously reported cases of eustachian valve endocarditis were secondary to staphylococcal infections: four cases related to intravenous drug use and one following staphylococcal pneumonia. This case is unusual as $E$ cloacae is present in normal intestinal flora. There was no evidence that the pacing wire was either an infected site or source. However, intermittent contact between the eustachian valve and pacing wire may have resulted in endothelial damage, predisposing the valve to infection.

P R JAMES D DAWSON S M C HARDMAN 